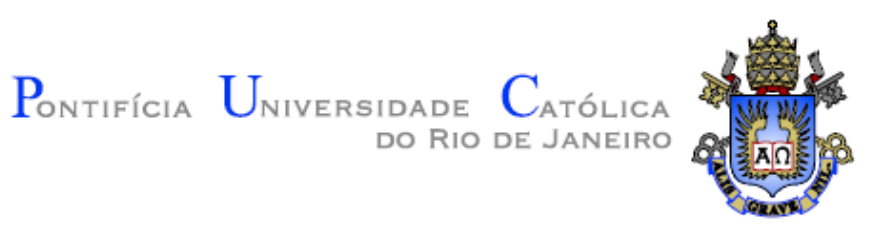

Bruno Henriques Dias

\title{
Programação Dinâmica Estocástica e Algoritmo de Fechos Convexos no Planejamento da Operação de Sistemas Hidrotérmicos
}

Tese apresentada como requisito parcial para obtenção do título de Doutor pelo Programa de Pós-Graduação em Engenharia Elétrica da PUC-Rio.

Orientadores: Reinaldo Castro Souza André Luís Marques Marcato 


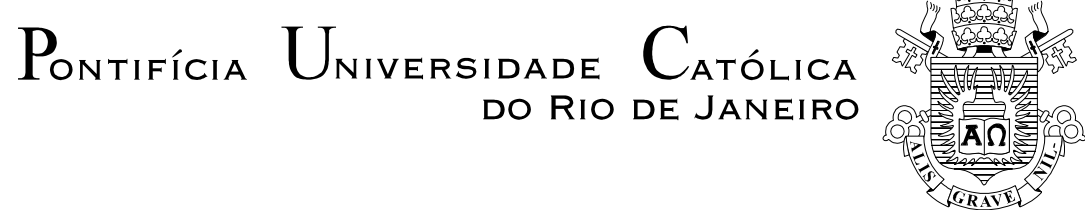

\section{Bruno Henriques Dias}

\section{Programação Dinâmica Estocástica e Algoritmo de Fechos Convexos no Planejamento da Operação de Sistemas Hidrotérmicos}

Tese apresentada como requisito parcial para obtenção do grau de Doutor pelo Programa de Pós-Graduação em Engenharia Elétrica do Departamento de Engenharia Elétrica do Centro Técnico Científico da PUC-Rio. Aprovada pela Comissão Examinadora abaixo assinada.

Prof. Reinaldo Castro Souza

Orientador

Departamento de Engenharia Elétrica - PUC-Rio

Prof. André Luís Marques Marcato

Co-orientador

UFJF

Prof. Edimar José de Oliveira UFJF

Prof. Edmarcio Antonio Belati

UFABC

Prof. Leonardo Lima Gomes

IAG/PUC-Rio

Prof. Ivo Chaves da Silva Junior UFJF

Prof. João Alberto Passos Filho

UFJF

Prof. José Eugenio Leal

Coordenador Setorial do Centro

Técnico Científico - PUC-Rio

Rio de Janeiro, 05 de julho de 2010 
Todos os direitos reservados. É proibida a reprodução total ou parcial do trabalho sem autorização da universidade, do autor e do orientador.

\section{Bruno Henriques Dias}

Bruno Henriques Dias é formado em Engenharia Elétrica pela Universidade Federal de Juiz de Fora - UFJF (2005) e obteve seu título de mestre em Engenharia Elétrica pela Pontifícia Universidade Católica do Rio de Janeiro - PUCRio (2007). Atualmente finaliza seu doutorado na mesma instituição. Tem atuado em pesquisa nas áreas de Planejamento da Operação de Sistemas Elétricos, Coordenação Hidrotérmica, Comercialização e Mercados de Energia Elétrica.

Ficha Catalográfica

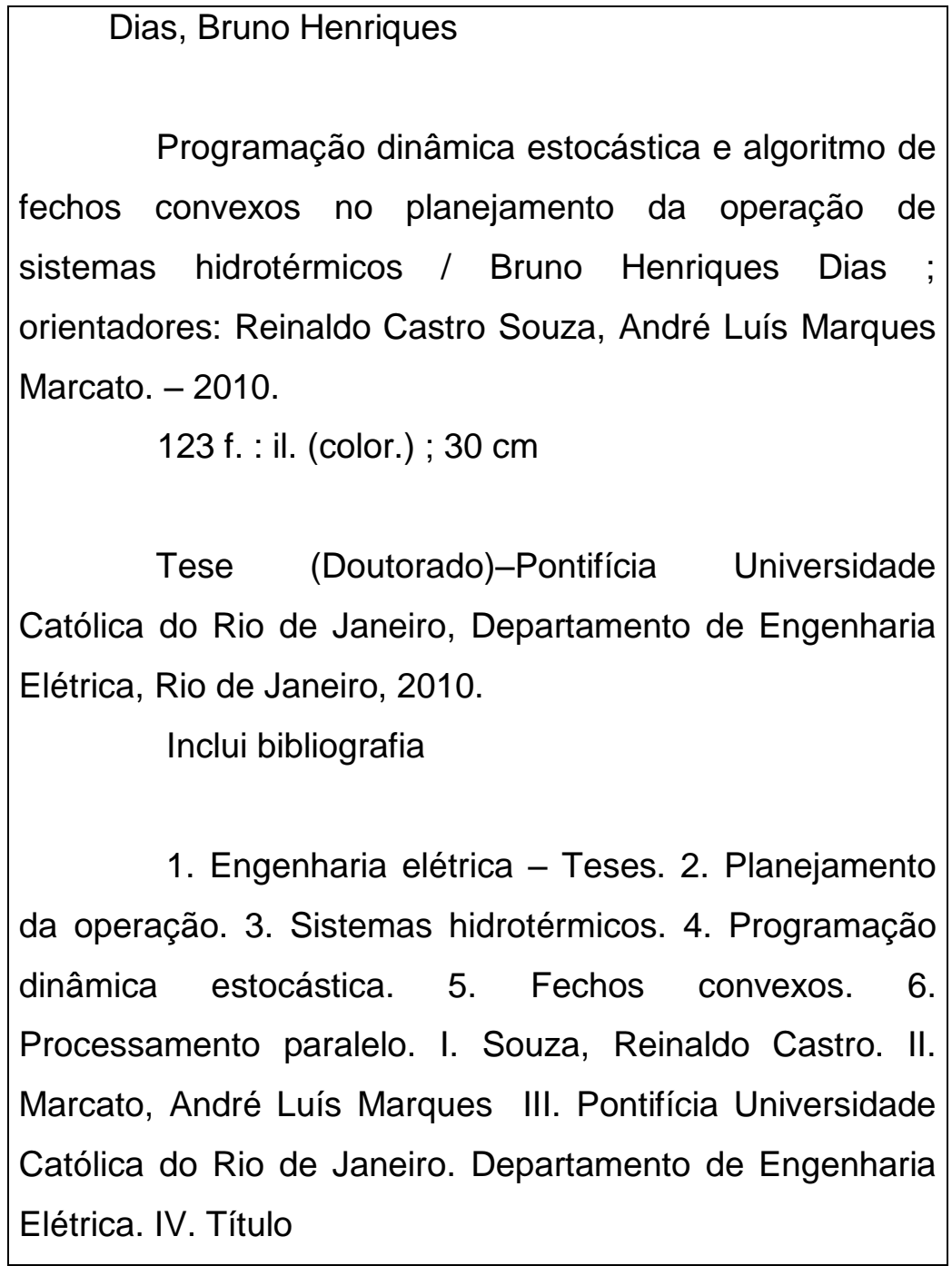

CDD: 621.3 
Dedico este trabalho àquela que foi a minha primeira professora, me ensinando as primeiras letras e me incentivando, ao longo dos anos, na busca constante pelo aprendizado: minha Mãe.

E ao meu Pai, que mesmo distante, encontra maneiras de dizer que está sempre por perto. 


\section{Agradecimentos}

"Agradecer é reconhecer que não conquistamos nada sozinhos"

Agradeço inicialmente e, sobretudo, a Deus, pela vida, oportunidade constante de aprendizado.

Ao amor, exemplo e dedicação incondicional de minha mãe, Regina, que tornou possível a realização deste trabalho. $E$ aos bons momentos vividos ao lado de meu pai, que me inspiram e trazem felizes recordações.

A minha querida irmã, Fernanda, amiga de todos os momentos.

Agradeço eternamente o carinho e a atenção de D. Isabel Salomão, por todos os ensinamentos e pela força, nos momentos mais difíceis. $E$ aos amigos da Comunidade Espírita "A Casa do Caminho" pelos laços de amizade fraternos.

A minha noiva, Anne Karole, por todo amor e apoio de sempre.

A toda minha família, fonte eterna de carinho e inspiração, em especial pelo auxílio de meus tios Itamar Henriques e Elimar Dias. Agradeço sempre ao Roberto Dias e Marcílio Henriques, meus primos e eternos amigos, companheiros de ideal no aprendizado constante.

Meus sinceros agradecimentos aos muitos amigos que me incentivam na minha caminhada. Agradeço em especial, ao Ivo Junior, Bruno Beloti e Leonardo Moraes, que me incentivaram, cada um em um momento diferente, no desenvolvimento deste trabalho.

Dedico minha especial gratidão ao amigo Murilo Soares, pelo apoio, discussões, e principalmente pelas idéias, durante todo o doutorado. Meu reconhecimento de que a realização deste trabalho só foi possível devido à sua ajuda.

Agradeço também ao Tales Ramos e Rafael Bruno, pelos muitos momentos de trabalho e estudo, além da amizade dividida durante todo o tempo de convivência além da participação neste trabalho. Agradeço ainda ao Filipe Niquini pelo tempo de excelente convivência e por seu constante apoio e ao Marcelo Tomim, pelas discussões e auxílio em parte da tese.

Aos professores Edimar Oliveira, Marley Velasco e Mônica Barros, Edmarcio Belati, Leonardo Gomes, Ivo Chaves Junior e João Alberto pela participação e pelas sugestões na proposta de tese e na defesa de tese.

Aos meus orientadores, Reinaldo Souza e André Marcato, que me possibilitaram a realização deste trabalho, concedendo a oportunidade de continuar no doutorado, além de todo o apoio no desenvolvimento do mesmo.

A todos os amigos que, apesar de não estarem nominalmente citados, me incentivaram com suas amizades e com a torcida para que eu vencesse mais este desafio, meus mais sinceros agradecimentos.

A CAPES e ao CNPq pelo apoio financeiro recebido durante a realização deste trabalho. 


\section{Resumo}

Henriques Dias, Bruno; Castro Souza, Reinaldo Programação Dinâmica Estocástica e Algoritmo de Fechos Convexos no Planejamento da Operação de Sistemas Hidrotérmicos. Rio de Janeiro, 2010. 123p. Tese de Doutorado - Departamento de Engenharia Elétrica, Pontifícia Universidade Católica do Rio de Janeiro.

Esta tese apresenta uma nova proposta para modelagem das funções de custo futuro, utilizadas na Programação Dinâmica Estocástica (PDE). A técnica proposta é aplicada ao planejamento da operação de médio prazo de sistemas elétricos de potência. Através da discretização do espaço de estados, o algoritmo de fechos convexos (convex hull) é utilizado na obtenção de uma série de hiperplanos que compõe um conjunto convexo. Estes planos representam uma aproximação linear por partes da função de custo futuro. O custo operacional médio utilizando a metodologia proposta considerando-se um único cenário de afluências foi comparado com o custo obtido da programação dinâmica dual determinística para o mesmo cenário de afluências. Esta análise mostra a convergência das duas metodologias e é utilizada para determinar o nível mínimo de discretização necessário para modelagem das funções de custo futuro. A partir deste resultado é feita a extensão da análise para diversos cenários de afluências utilizando-se a metodologia proposta, sendo a função de custo futuro obtida através da média do custo de operação para os diversos cenários, em cada discretização. A aplicabilidade do método é mostrada utilizando um caso exemplo de duas usinas hidrelétricas reais em cascata. Adicionalmente, um estudo de caso analisa as vantagens da paralelização do código de programação, onde métricas tais como fator de aceleração e eficiência são analisadas. Por fim, é apresentada uma simulação contendo todo o sistema elétrico brasileiro, representado por reservatórios equivalentes.

\section{Palavras-chave}

Planejamento da Operação, Sistemas Hidrotérmicos, Programação Dinâmica Estocástica, Fechos Convexos, Processamento Paralelo. 


\section{Abstract}

Henriques Dias, Bruno; Castro Souza, Reinaldo Stochastic Dynamic Programming and Convex Hull Algorithm in the Hydrothermal Systems Operation Planning. Rio de Janeiro, 2010. 123p. PhD. Thesis Departamento de Engenharia Elétrica, Pontifícia Universidade Católica do Rio de Janeiro.

This thesis presents a new approach for the expected-cost-to-go functions modeling used in the stochastic dynamic programming (SDP) algorithm. The proposed technique is applied to the long-term operation planning of electrical power systems. Using state space discretization, the convex hull algorithm is used for constructing a series of hyperplanes that composes a convex set. These planes represent a piecewise linear approximation for the expected-costto-go functions. The mean operation costs obtained by the proposed methodology for a single water inflow scenario were compared with those from the deterministic dual dynamic programming for the same inflow scenario.This sensitivity analysis shows the convergence of both methods and is used to determine the minimum discretization level necessary to model the expectedcost-to-go functions. From the obtained result the proposed methodology is extended to the analysis of a set of water inflow scenarios, where the expectedcost-to-go function is obtained by the mean operation cost to all the considered scenario in each discretization level. The applicability of the proposed methodology for two hydro plants in a cascade is demonstrated. Additionally, a case study using code parallelization is presented aiming at gaining computational performance, where the parallelization performance, as speedup and efficiency are measured. To finish with a simulation with the whole Brazilian electrical system considering aggregated reservoir is presented.

\section{Keywords}

Operation Planning, Hydrothermal Systems, Stochastic Dynamic Programming, Convex Hull, Parallel Processing. 


\section{Sumário}

1 Introdução 15

1.1. Breve Histórico do Planejamento Energético no SEB 16

1.2. Objetivo 19

1.3. Publicações e Submissões Decorrentes da Pesquisa 19

1.4. Organização da Tese 20

2 Estado da Arte em Planejamento da Operação de Sistemas Hidrotérmicos 22

2.1. Planejamento de Sistemas Hidrotérmicos 22

2.2. Planejamento da Operação de Médio Prazo 25

2.3. Modelo de Reservatórios Equivalentes de Energia 29

2.4. Revisão Bibliográfica 33

2.4.1. Planejamento de Longo/Médio Prazo 34

2.4.2. Planejamento de Curto Prazo 36

2.4.3. Técnicas de Processamento Paralelo 38

2.4.4. Técnicas de Inteligência Artificial 40

2.4.5. Sistemas de Suporte à Decisão 47

2.4.6. Programação Dinâmica 48

3 Programação Dinâmica Estocástica e Algoritmo de Fechos Convexos 52

3.1. Introdução 52

3.2. Programação Dinâmica Estocástica - Formulação do Problema 53

3.2.1. Função Objetivo 54

3.2.2. Restrições 54

3.3. Modelagem das Funções de Custo Futuro 56

3.3.1. Introdução 56

3.3.2. Exemplo Didático 56

3.3.3. Método Proposto: Conjuntos Convexos 64

3.3.4. Eliminação de Planos $\quad 69$

4 Computação Paralela $\quad 72$ 
4.1. Introdução 72

4.2. Clusters de Computadores $\quad 74$

4.3. Métricas de Desempenho em Sistemas Paralelos $\quad 75$

4.3.1. Fator de Aceleração $\quad 75$

4.3.2. Fator de Aceleração Máximo $\quad 76$

4.3.3. Eficiência 76

4.4. Interfaces de Programação Paralela $\quad 77$

4.4.1. Message Passing Interface (MPI) 77

4.4.2. Open Multi-Processing Application Program Interface (OpenMP) 78

4.5. Proposta de Paralelização do Problema de Planejamento Energético 79

5 Simulações e Resultados $\quad 80$

5.1. Introdução 80

5.2. Sistema Tutorial: obtenção das funções de custo futuro 80

5.3. Estudo de Caso 1: Sistema com dois reservatórios 88

5.3.1. Análise de Sensibilidade 90

5.3.2. Modelagem das Funções de Custo Futuro 9

5.3.3. Simulação com pós-estudo utilizando cenário médio 94

5.4. Estudo de Caso 2: processamento paralelo 96

5.5. Estudo de Caso 3: Sistema Elétrico Brasileiro 98

5.5.1. Simulação dos subsistemas Sul e Sudeste/Centro-Oeste 99

5.5.2. Simulação dos SEB completo 103

6 Conclusões 104

6.1. Conclusão 104

6.2. Trabalhos Futuros 105

7 Referências Bibliográficas 107 


\section{Lista de Figuras}

Figura 1: Complementaridade hidrológica dos subsistemas

Figura 2: Modelagem dos sistemas hidrotérmicos no planejamento da operação

24

Figura 3: Representação da decisão operativa de um sistema hidrotérmico 28

Figura 4: Função de custo imediato, custo futuro e custo total (SILVA, 2001) 28

Figura 5: Sistema equivalente de energia 30

Figura 6: Sistema contendo sete usinas 31

Figura 7: Representação do sistema utilizado no exemplo didático 57

Figura 8:Aproximação da FCF construída no estágio $3 \quad 61$

Figura 9: Aproximação da FCF construída no estágio 2

Figura 10: Aproximação da FCF construída no estágio 1

Figura 11: Exemplo de Conjuntos Convexo e Não-convexo 64

Figura 12: Pontos aleatórios no plano bidimensional 65

Figura 13: Algoritmo QuickHull - passo 1

Figura 14: Algoritmo QuickHull - passo 2

Figura 15: Algoritmo QuickHull - passo $3 \quad 66$

Figura 16: Algoritmo QuickHull - passo $4 \quad 67$

Figura 17: Conjunto convexo obtido pelo algoritmo QuickHull 67

Figura 18: Conjunto convexo no plano tridimensional: (a) conjunto aleatório de pontos e (b) Conjunto convexo obtido 68

Figura 19: Algoritmo da obtenção das funções de custo futuro utilizando fechos convexos. 69

Figura 29: Conjunto convexo obtido a partir dos custos calculados 70

Figura 30: Conjunto convexo com pontos artificiais. 70

Figura 31: Conjunto de planos de corte que aproximam a FCF 71

Figura 20: Cluster de computadores tipo Beowulf genérico. 74

Figura 21: Algoritmo da obtenção das funções de custo futuro utilizando fechos convexos e processamento paralelo $\quad 79$

Figura 22: níveis de discretização do reservatório 81

Figura 23: Conjunto convexo do estágio três 83 
Figura 24: Função de custo futuro aproximada obtida no terceiro estágio. $\quad 83$

Figura 25: Função de custo futuro aproximada obtida no segundo estágio. 85

Figura 26: Função de custo esperado obtido no primeiro estágio 86

Figura 27: Aproximação da função de custo futuro para diferentes discretizações

Figura 28: Custos operativos do sistema com reservatório único 87

Figura 32: Diagrama esquemático das duas usinas do SEB utilizadas. 88

Figura 33: Mercado de energia considerado $\quad 89$

Figura 34: Afluência incremental à usina de Sobradinho 91

Figura 35: função de custo futuro obtida no estágio 60

Figura 36: função de custo futuro obtida no estágio 59

Figura 37: função de custo futuro obtida no estágio 2

Figura 38: Geração hídrica e térmica 95

Figura 39: Energia armazenada no reservatório de Sobradinho 95

Figura 40: Energia armazenada no reservatório de itaparica 96

Figura 41: Geração hídrica e térmica do caso em estudo 97

Figura 42: Fator de aceleração do algoritmo paralelo 98

Figura 43: Eficiência do algoritmo paralelo. 98

Figura 44: Função de custo futuro obtida no último estágio 99

Figura 45: Função de custo futuro obtida no estágio 59

Figura 46: Função de custo futuro obtida no estágio 2

Figura 47: Geração hidráulica e térmica do caso em estudo 101

Figura 48: Gráfico de armazenamento dos Subsistemas. 101

Figura 49: Fator de aceleração do algoritmo paralelo 102

Figura 50: Eficiência do algoritmo paralelo. 102

Figura 51: Geração hidráulica e térmica do SEB 103 


\section{Lista de tabelas}

Tabela 1: Referências de planejamento de longo/médio prazo 36

Tabela 2: Referências de planejamento de curto prazo 37

Tabela 3: Referências de processamento paralelo 40

Tabela 4: Referências de inteligência artificial no planejamento energético 46

Tabela 5: Referências de sistemas de suporte à decisão 48

Tabela 6: Referências de programação dinâmica 50

Tabela 7: Dados da usina hidrelétrica São Simão (ONS) 57

Tabela 8: Dados das usinas termelétricas $\quad 57$

Tabela 9: Cenário de afluências ao reservatório São Simão 58

Tabela 10: Volume do reservatório em relação às discretizações 58

Tabela 11: Decisões térmicas possíveis do caso exemplo 59

Tabela 12: Resultado dos cálculos da PDE no terceiro estágio 61

Tabela 13: Decisões térmicas no estágio $2\left(x_{t}=50 \%, y_{t}=\right.$ alta $)$

Tabela 14: Resultado dos cálculos da PDE no segundo estágio 62

Tabela 15: Resultado dos cálculos da PDE no primeiro estágio 63

Tabela 16: Características operativas da usina hidrelétrica 80

Tabela 17: Características operativas das usinas termelétricas 81

Tabela 18: Cenários de afluências considerados 81

Tabela 19: custos esperados do terceiro estágio 82

Tabela 20: Número de retas de corte obtidas no último estágio 84

Tabela 21: custos esperados do segundo estágio 84

Tabela 22: custos esperados do primeiro estágio 85

Tabela 23: Dados da usinas hidrelétricas consideradas. 88

Tabela 24: Dados operativos das usinas termelétricas $\quad 89$

Tabela 25: Demanda considerada para os 5 anos do estudo (MW-Médio) 89

Tabela 26: comparação de resultados para diferentes níveis de discretização 90

Tabela 27: Custo esperado para três cenários de afluências 93

Tabela 28: Tempo total gasto em função do número de processadores utilizados

Tabela 29: Tempo de processamento 


\section{Lista de Abreviaturas}

AG

CEPEL

EAD

$\mathrm{EAR}_{\max }$

$\mathrm{EBH}$

EE

ENA

EPE

FCF

$\mathrm{FCl}$

FCT

FLOPS

FR

IA

LA

LP

MLP

MLT

MPI

ONS

PAR
Algoritmo Genético

Centro de Pesquisas de Energia Elétrica

Equação de Atendimento à Demanda

Energia Armazenável Máxima

Equação de Balanço Hídrico

Estratégia Evolutiva

Energia Natural Afluente

Empresa de Pesquisa Energética

Função de Custo Futuro

Função de Custo Imediato

Função de Custo Total

Floating Point Operations per Second-

número de operações de ponto flutuante por segundo

Fluxo em Rede

Inteligência Artificial

Lagrangeano Aumentado

Lista de Prioridade

Multi-Layer Perceptron

Média de Longo Termo

Message Passing Interface

Operador Nacional do Sistema Elétrico

Auto-regressivo Periódico (do inglês) 


\begin{tabular}{|c|c|}
\hline PC & Computador Pessoal (do inglês) \\
\hline PD & Programação Dinâmica \\
\hline PDAS & Programação Dinâmica por Aproximações Sucessivas \\
\hline PDD & Programação Dinâmica Discreta \\
\hline PDDD & Programação Dinâmica Dual Determinística \\
\hline PDDE & Programação Dinâmica Dual Estocástica \\
\hline PDE & Programação Dinâmica Estocástica \\
\hline PDEA & Programação Dinâmica Estocástica Amostral \\
\hline PDI & Programação Dinâmica Incremental \\
\hline PIM & Programação Inteira Mista \\
\hline PL & Programação Linear \\
\hline PNL & Programação Não Linear \\
\hline PPL & Problema de Programação Linear \\
\hline PVM & Parallel Virtual Machine \\
\hline $\mathrm{RL}$ & Relaxação Lagrangeana \\
\hline RNA & Rede Neural Artificial \\
\hline S & Speedup - Fator de aceleração \\
\hline SA & Simulated Annealing - recozimento simulado \\
\hline SEB & Sistema Elétrico Brasileiro \\
\hline SIN & Sistema Interligado Nacional \\
\hline SOM & Self-Organized Map - Mapa auto-organizável \\
\hline VAF & Volume Armazenado Final \\
\hline OPENMP & Open Multi-Processing Application Program Interface \\
\hline
\end{tabular}

